\title{
Design of Tracking Control System by Closed-Loop Pole Placement
}

\author{
Shengguo Zhang ${ }^{1,}$, Shuo Zhang ${ }^{2, b}$ and Guoheng Zhang ${ }^{1, c}$ \\ ${ }^{1}$ School of Electrical Engineering, Northwest University for Nationalities, Lanzhou, 730030, China \\ ${ }^{2}$ School of Automation, Hangzhou Dianzi University, Hangzhou, 310018, China

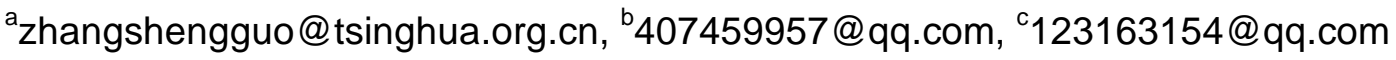

Keywords: Pole placement, Deviation integrator, State augmentation, Steady state error.

Abstract: This paper focuses on designing the MIMO tracking control system which can achieve zero steady state errors and obtain desired dynamic performances, simultaneously. By using state feedback, the closed-loop stability and desired dynamic performances are guaranteed. By integrating the deviations between reference inputs and measured outputs, i.e. augmenting system states, the steady state errors are eliminated. The state feedback gain and the forward integral gain are derived synchronously based on closed-loop pole placement method. Studying results indicate the closed- loop system achieves zero steady state errors and obtains desired dynamic performances as well as closed-loop stability. By pole placement of the closed-loop system combining forward integrator with state feedback controller, a complete tracking control system can be designed.

\section{Introduction}

For many control systems such as servo or following systems, they require the system outputs must track the system inputs precisely. This implies the steady state error of closed-loop system has to be zero. At the same time, a tracking control system must guarantee its closed-loop stability as well as obtain desired dynamic performances [1]. The steady state error can be eliminated or decreased by introducing integrators and increasing forward integral gain. State feedback or output feedback can be used to guarantee the system stability and dynamic performances [2]. The forward integrator and the state feedback controller should be designed synthetically so that the different control requirements can be met simultaneously [3].

In this paper, a tracking control system [4], which has different requirements of stability, dynamic performances, and especially steady state performances, are designed. The stability and the dynamic performances are guaranteed by state feedback with appropriate gain. The steady state performances are achieved by series integrator with appropriate forward gain, which is the integral of deviation between reference input and fedback output. And the forward integral gain and the state feedback gain are determined synchronously by closed-loop pole placement based on state space model.

\section{Tracking Control System and Its Designing Theories}

A multi-input-multi-output (MIMO) control system can be represented by state space model as:

$$
\left\{\begin{array}{l}
\{(t)=\boldsymbol{A} x(t)+\boldsymbol{B} u(t)+d(t) \\
y(t)=\boldsymbol{C} x(t)
\end{array}\right.
$$

where $x(t)$ is $\mathrm{n}$-dimension state vector, $u(t)$ is m-dimension control input vector, and $y(t)$ is $\mathrm{r}$-dimension output vector, and $d(t)$ is n-dimension disturbance input vector, and $\boldsymbol{A}, \boldsymbol{B}, \boldsymbol{C}$ are all constant matrixes with appropriate dimensions. Now a MIMO tracking control system is under consideration. Given the r-dimension reference input vector is $q(t)$, thus, the control tasks are finding an appropriate control input $u(t)$, which can make the closed-loop system meet following requirements while the disturbance input $d(t)$ is acting on the system: 1) the closed-loop system is asymptotically stable;2) the closed-loop system has desired dynamic performances; 3 ) the system output $y(t)$ can track the reference input $q(t)$ with zero steady state error.

State Augmentation. Defining deviation vector $e(t)$ as: 


$$
e(t)=y(t)-q(t)
$$

and introducing the integral vector $\bar{x}(t)$ of deviation vector $e(t)$, which has the same dimension as output vector $y$ and its transpose is:

$$
\overline{\boldsymbol{x}}^{\mathrm{T}}(t)=\left[\begin{array}{llll}
\bar{x}_{1}(t) & \bar{x}_{2}(t) & \mathrm{K} & \bar{x}_{\mathrm{r}}(t)
\end{array}\right]=\left[\int_{0}^{t} e_{1}(\tau) \mathrm{d} \tau \quad \int_{0}^{t} e_{2}(\tau) \mathrm{d} \tau \quad \mathrm{K} \quad \int_{0}^{t} e_{\mathrm{r}}(\tau) \mathrm{d} \tau\right]
$$

Thus, new augmented state vector $\bar{x}(t)$ is derived and its equation of motion can also be derived from Eq. 1 to Eq. 3:

$$
\mathcal{f}(t)=e(t)=y(t)-q(t)=\boldsymbol{C} x(t)-q(t),
$$

then the augmented system can be represented as following state space model:

$$
\left\{\begin{array}{l}
{\left[\begin{array}{l}
(t) \\
(t)
\end{array}\right]=\left[\begin{array}{ll}
\boldsymbol{A} & 0 \\
\boldsymbol{C} & 0
\end{array}\right]\left[\begin{array}{l}
x(t) \\
\bar{x}(t)
\end{array}\right]+\left[\begin{array}{l}
\boldsymbol{B} \\
0
\end{array}\right] u(t)+\left[\begin{array}{c}
d(t) \\
-q(t)
\end{array}\right] .} \\
y(t)=\left[\begin{array}{ll}
\boldsymbol{C} & 0
\end{array}\right]\left[\begin{array}{l}
x(t) \\
\bar{x}(t)
\end{array}\right]
\end{array}\right.
$$

Proving the Existence and Condition of Feedback Control Strategy. Supposing there is a state (including augmented state) feedback control strategy:

$$
u(t)=-\left[\begin{array}{ll}
\boldsymbol{K}_{1} & \boldsymbol{K}_{2}
\end{array}\right]\left[\begin{array}{l}
x(t) \\
\bar{x}(t)
\end{array}\right]=-\boldsymbol{K}_{1} x(t)-\boldsymbol{K}_{2} \bar{x}(t),
$$

where $\boldsymbol{K}_{1}$ and $\boldsymbol{K}_{2}$ are feedback gain vector of n-dimension and forward gain vector of r-dimension, respectively. If this feedback control strategy is effective, it has to enable the following closed-loop system whose steady state error is zero and which is asymptotically stable:

$$
\left[\begin{array}{l}
\hat{(}(t) \\
\hat{f}(t)
\end{array}\right]=\left[\begin{array}{cc}
\boldsymbol{A}-\boldsymbol{B} \boldsymbol{K}_{1} & -\boldsymbol{B} \boldsymbol{K}_{2} \\
\boldsymbol{C} & 0
\end{array}\right]\left[\begin{array}{l}
x(t) \\
\bar{x}(t)
\end{array}\right]+\left[\begin{array}{c}
d(t) \\
-q(t)
\end{array}\right] .
$$

Firstly, using Laplace transformation to Eq. 7, there can get:

$$
\left[\begin{array}{c}
X(s) \\
\bar{X}(s)
\end{array}\right]=\left(s \boldsymbol{I}-\left[\begin{array}{cc}
\boldsymbol{A}-\boldsymbol{B} \boldsymbol{K}_{1} & -\boldsymbol{B} \boldsymbol{K}_{2} \\
\boldsymbol{C} & 0
\end{array}\right]\right)^{-1}\left[\begin{array}{c}
D(s) \\
-Q(s)
\end{array}\right],
$$

where $I$ is a unit matrix with $(\mathrm{n}+\mathrm{r})$ dimension and $s$ is complex variable factor. Then giving the disturbance input $d(t)$ to be step signal $d_{0} \cdot 1(t)$ and the reference input $q(t)$ also to be step signal $q_{0} \cdot 1(t)$. And then using the final value theorem of Laplace transformation there can yield:

$$
\begin{aligned}
\lim _{t \rightarrow \infty}\left[\begin{array}{l}
x(t) \\
\bar{x}(t)
\end{array}\right]=\lim _{s \rightarrow 0} s\left[\begin{array}{c}
X(s) \\
\bar{X}(s)
\end{array}\right] & =\lim _{s \rightarrow 0} s\left(s \boldsymbol{I}-\left[\begin{array}{cc}
\boldsymbol{A}-\boldsymbol{B} \boldsymbol{K}_{1} & -\boldsymbol{B} \boldsymbol{K}_{2} \\
\boldsymbol{C} & 0
\end{array}\right]\right)^{-1}\left[\begin{array}{c}
d_{0} / s \\
-q_{0} / s
\end{array}\right], \\
& =-\left[\begin{array}{cc}
\boldsymbol{A}-\boldsymbol{B} \boldsymbol{K}_{1} & -\boldsymbol{B} \boldsymbol{K}_{2} \\
\boldsymbol{C} & 0
\end{array}\right]^{-1}\left[\begin{array}{c}
d_{0} \\
-q_{0}
\end{array}\right]
\end{aligned}
$$

From Eq. 9 there can be observed that $x(t)$ and $\bar{x}(t)$ are both tending to constants while $t$ is tending to infinity. It means $\delta(t)$ and $\delta(t)$ are both tending to zero while $t$ is tending to infinity. In accordance with Eq. 4, there can get:

$$
\lim _{t \rightarrow \infty} \mathcal{E}(t)=\lim _{t \rightarrow \infty} e(t)=\lim _{t \rightarrow \infty}[y(t)-q(t)]=0
$$


it indicates the steady state error of closed-loop system is zero and system outputs can track reference inputs precisely.

Secondly, In order to guarantee the closed-loop system to be asymptotically stable, all of eigenvalues of augmented system matrix $\bar{A}$ have to be on the left half of complex plane:

$$
\bar{A}=\left[\begin{array}{cc}
\boldsymbol{A}-\boldsymbol{B} \boldsymbol{K}_{1} & -\boldsymbol{B} \boldsymbol{K}_{2} \\
\boldsymbol{C} & 0
\end{array}\right] .
$$

These eigenvalues of system matrix $\bar{A}$ are completely dependent upon the selections of feedback gain $\boldsymbol{K}_{1}$ and forward gain $\boldsymbol{K}_{2}$. Once $\boldsymbol{K}_{1}$ and $\boldsymbol{K}_{2}$ are selected appropriately, the closed-loop system is not only asymptotically stable, but also can obtain desired dynamic performances.

Solving Feedback Gain $K_{1}$ and Forward Gain $K_{2}$ by Pole Placement Method. Fig. 1 is shown the control structure of the tracking system represented by Eq. 5, whose states are augmented due to introduction of the integrator.

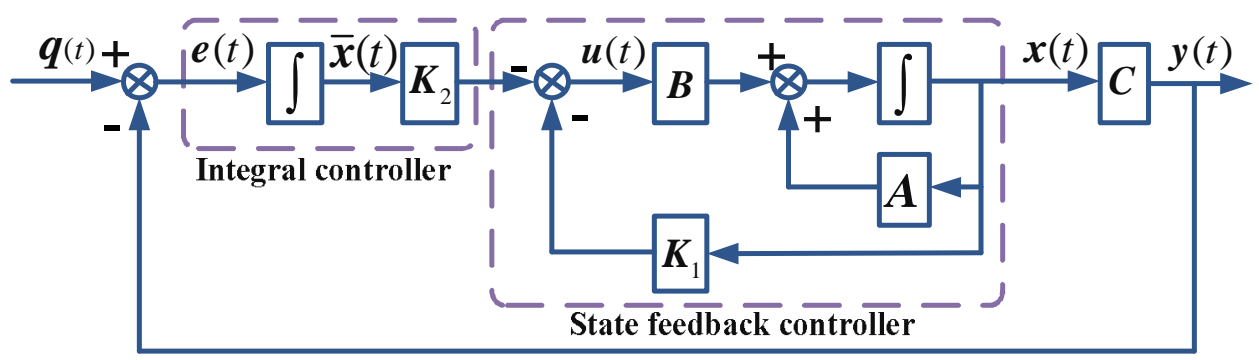

Fig. 1 The control structure of the tracking system whose states are augmented

According to the state space theory [5], if a system can be assigned arbitrary closed-loop poles via state feedback, only when this closed-loop system is of state controllability. So for augmented system shown in Fig. 1 and represented by Eq. 5, its states (including augmented states) have to be controllable. Here gives a theorem without proof to judge the controllability of an augmented system. This theorem can be summarized as follows:

A system represented by Eq. 1 is augmented to be another system represented by Eq. 5. The augmented system will be controllable if and only if the original system is controllable and:

$$
\operatorname{rank}\left(\left[\begin{array}{cc}
A & B \\
C & 0
\end{array}\right]\right)=\mathrm{n}+\mathrm{r}, \mathrm{m} \geq \mathrm{r}, \operatorname{rank}(\boldsymbol{C})=\mathrm{r} \text {. }
$$

After the state controllability of the augmented system is determined, the closed-loop pole placement method can be used to solve the state feedback gain $\boldsymbol{K}_{1}$ and forward integral gain $\boldsymbol{K}_{2}$.

First assuming that:

$$
\boldsymbol{K}_{1}=\left[\begin{array}{llll}
k_{11} & k_{12} & \mathrm{~L} & k_{1 \mathrm{n}}
\end{array}\right], \quad \boldsymbol{K}_{2}=\left[\begin{array}{llll}
k_{21} & k_{22} & \mathrm{~L} & k_{2 \mathrm{r}}
\end{array}\right],
$$

then solving the eigenpolynomial of the augmented system:

$$
\begin{aligned}
f(\boldsymbol{\lambda}) & =\operatorname{det}(\overline{\boldsymbol{A}})=|\lambda \boldsymbol{I}-\overline{\boldsymbol{A}}|=\mid \lambda \boldsymbol{I}-\left[\begin{array}{cc}
\boldsymbol{A}-\boldsymbol{B} \boldsymbol{K}_{1} & -\boldsymbol{B} \boldsymbol{K}_{2} \\
\boldsymbol{C} & 0
\end{array}\right], \\
& =a_{\mathrm{n}+\mathrm{r}-1} \lambda^{\mathrm{n}+\mathrm{r}}+a_{\mathrm{n}+\mathrm{r}-2} \lambda^{\mathrm{n}+\mathrm{r}-1}+\mathrm{L}+a_{1} \lambda+a_{0}
\end{aligned}
$$

where $a_{0}, a_{1}, \ldots, a_{\mathrm{n}+\mathrm{r}-2}$ and $a_{\mathrm{n}+\mathrm{r}-1}$ are dependent upon $k_{11}, k_{12}, \ldots, k_{1 \mathrm{n}}$ and $k_{21}, k_{22}, \ldots k_{2 \mathrm{r}}$ completely. And then listing the eigenpolynomial of the augmented system according to desired closed-loop poles $\lambda_{1}$, $\lambda_{2}, \ldots, \lambda_{n+r}$ which are given or solved on the basis of dynamic performance requirements: 


$$
\begin{aligned}
f^{*}(\lambda) & =\left(\lambda-\lambda_{1}\right)\left(\lambda-\lambda_{2}\right) \mathrm{L}\left(\lambda-\lambda_{\mathrm{n}+\mathrm{r}}\right) \\
& =b_{\mathrm{n}+\mathrm{r}-1} \lambda^{\mathrm{n}+\mathrm{r}}+b_{\mathrm{n}+\mathrm{r}-2} \lambda^{\mathrm{n}+\mathrm{r}-1}+\mathrm{L}+b_{1} \lambda+b_{0},
\end{aligned}
$$

Last, let $f(\lambda)=f^{*}(\lambda)$ and there yields an algebraic equation set: $a_{0}=b_{0}, a_{1}=b_{1}, \ldots, a_{\mathrm{n}+\mathrm{r}-2}=b_{\mathrm{n}+\mathrm{r}-2}, a_{\mathrm{n}+\mathrm{r}-1}$ $=b_{\mathrm{n}+\mathrm{r}-1}$. Solving this equation set and the feedback gain $\boldsymbol{K}_{1}$ and forward gain $\boldsymbol{K}_{2}$ can be solved.

\section{Designing Example}

There is a servo system which has two inputs and two outputs. Control requirements are settle time less than 1 second, overshoot less than 5\%, and steady state error must be zero. The state space model of the system is as follows:

$$
\hat{\alpha}=\left[\begin{array}{rr}
0 & 1 \\
-3 & -4
\end{array}\right] x+\left[\begin{array}{ll}
1 & 0 \\
0 & 1
\end{array}\right] u, \quad y=\left[\begin{array}{ll}
1 & 2 \\
3 & 2
\end{array}\right] x .
$$

It can be verified this open system's state is controllable completely. Therefore, there must have a state feedback control strategy so that the closed-loop poles can be placed arbitrarily. These poles may as well be placed at $[-4,-5]$ (still many other points). Then the state feedback gain $\boldsymbol{K}$ can be solved easily and the state space model of closed-loop system is as follows:

$$
\mathcal{W}=(\boldsymbol{A}-\boldsymbol{B} \boldsymbol{K}) x=\left[\begin{array}{rr}
-4 & 0 \\
0 & -5
\end{array}\right] x, \quad y=\boldsymbol{C} x=\left[\begin{array}{ll}
1 & 2 \\
3 & 2
\end{array}\right] x .
$$

Fig. 2 is shown the unit step responses of this state feedback closed-loop system.

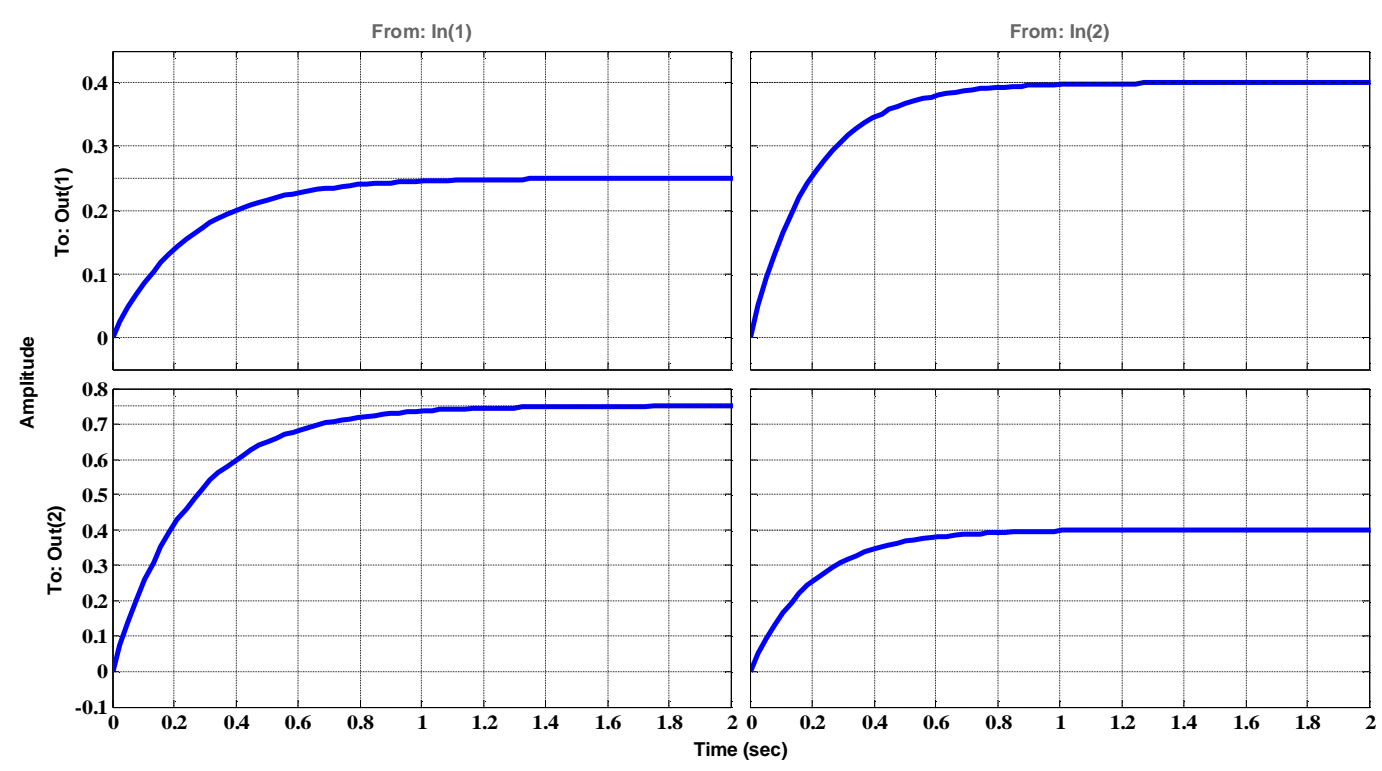

Fig. 2 The unit step responses of state feedback closed-loop system

There can be observed from Fig. 2, the steady state errors obviously are not zeros although the settle time is less than 1 second and the system has no overshoot. What's worse, the closed-loop system is coupled seriously between two outputs and two inputs.

The system's states are controllable as well as the system meets conditions that Eq. 12 represents. In order to eliminate the steady state errors and decouple the system, system's states are augmented and system is reconfigured as shown in Fig. 1 . The desired closed-loop poles are placed at $[-4,-5,-8,-10]$ by the method that Eq. 13 to Eq. 15 represent. Then the state feedback gain $\boldsymbol{K}_{1}$ and the forward integral gain $\boldsymbol{K}_{2}$ can be solved and the state space model of this state augmentation closed-loop system is as follows: 


$$
\begin{aligned}
& {\left[\begin{array}{l}
\& \\
\&
\end{array}\right]=\left[\begin{array}{cccc}
14.9820 & 1.5398 & -22.1004 & 23.9927 \\
-2.8970 & 8.0180 & 23.7915 & -7.7294 \\
1 & 2 & 0 & 0 \\
3 & 2 & 0 & 0
\end{array}\right]\left[\begin{array}{l}
x \\
\bar{x}
\end{array}\right]+\left[\begin{array}{rr}
1 & 0 \\
0 & 1 \\
-1 & 0 \\
0 & -1
\end{array}\right]\left[\begin{array}{l}
d \\
q
\end{array}\right] .} \\
& y=\left[\begin{array}{llll}
1 & 2 & 0 & 0 \\
3 & 2 & 0 & 0
\end{array}\right]\left[\begin{array}{l}
x \\
\bar{x}
\end{array}\right]
\end{aligned}
$$

Fig. 3 is shown the unit step responses of this state augmentation closed-loop system. There can be observed from Fig. 3, the steady state errors become to be zeros, at the same time, the settle time is less than 1 second and the system has no overshoot. Furthermore, the second output response to the first input and the first output response to the second input both tend to zeros within the settle time. This means the closed-loop system is decoupled completely.

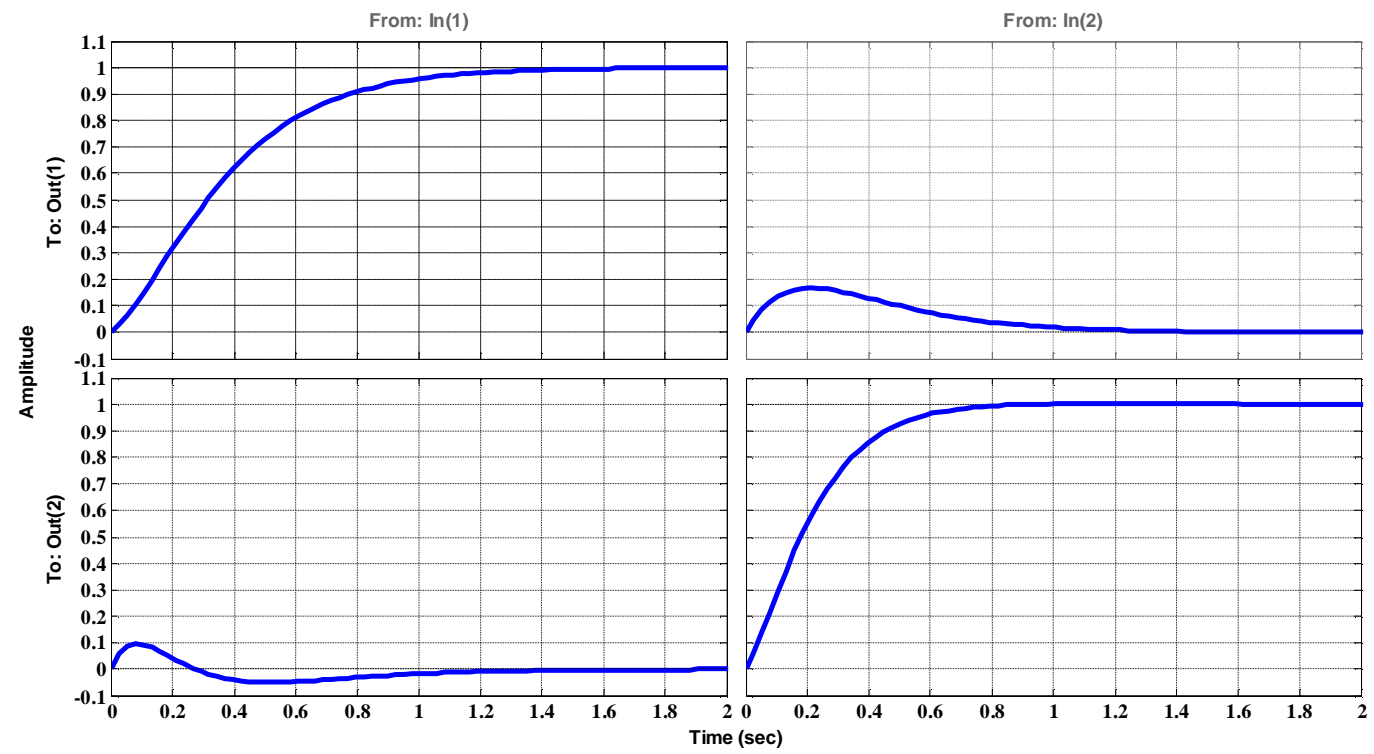

Fig. 3 The unit step responses of state augmentation closed-loop system

\section{Conclusions}

By designing state feedback controller, the stability and dynamic performances of the closed-loop system can be guaranteed. Yet there yields steady state errors which are unallowable for tracking control system. In order to eliminate these steady state errors, system states are augmented. The augmented states are integrals of deviations between reference inputs and measured outputs. The state feedback gain and the forward integral gain are obtained simultaneously via closed-loop pole placement. Thus the complete closed-loop control laws of a tracking control system, which can not only guarantee the stability and desired dynamic performances but also achieve zero steady state errors, are designed.

\section{Acknowledgements}

This work was financially supported by National Natural Science Foundation of China (51465053) and Fundamental Research Funds for the Central Universities (31920140082).

\section{References}

[1] Shousong Hu, Automatic Control Principle, fifth ed., Science Press, Beijing 2007.

[2] Li Yu, Modern Control Theory, Tsinghua University Press, Beijing 2007. 
[3] Xueyan Zhao, Feiqi Deng, Divided State Feedback Control of Stochastic Systems, IEEE T. Automat. Contr., 2015, 60(7)1870-1885.

[4] Shengguo Zhang, Yu Zhu, Haihua Mu, et al., Decoupling and Levitation Control of a 6-DOF Magnetically Levitated Stage with Moving Coils Based on Commutation of Coil Array, P I Mech. ENG. I-J. SYS., 2012, 226(7)875-886.

[5] Siying Zhang, Liqun Gao, Modern Control Theory, Tsinghua University Press, Beijing 2006. 